\title{
TIME RESOLVED SPECTROSCOPY OF AM CVn
}

\author{
C. Lázaro ${ }^{1}$, J.-E. Solheim ${ }^{2}$ and M.J. Arévalo ${ }^{1,3}$ \\ 1- Instituto de Astrofisica de Canarias, Spain \\ 2- University of Tromsø, Norway \\ 3- Dpto. Fisica, Universidad Politecnica de Canarias, Spain
}

\section{ABSTRACT}

The preliminary results of time resolved spectroscopy of the He White Dwarf system AM CVn are presented. We have searched for spectral variations at different photometric periods. These are found at different time scales although this does not particularly favour any of the searched periods.

\section{INTRODUCTION}

AM CVn (HZ 29) is a system whose photometric light curve shows variable minima (usually with a double hump) and (U-B), (B-V) color changes, with a periodicity of about 18 minutes (Smak, 1967; Warner and Robinson, 1972; Krzeminski, 1971). Rapid flickering, typical of the cataclysmic variables, has also been observed (Warner and Robinson, 1972).

In its optical spectra there are no hydrogen 1ines, and only broad asymmetric absorption lines of He I are observed. These lines are wider and shallower than in ordinary BD White Dwarfs (Robinson and Faulkner, 1972). The line profiles may be partly filled up by emission cores with long term variations (Voikhanskaya, 1982).

Among different models proposed over the years, a model with two helium white dwarfs orbiting each other as proposed by Faulkner et a1. (1972) is the most plausible. In this model a low mass lobe-filling white dwarf transfers mass to a more luminous primary - and an optical thick disk with a considerable wind outflow is created (Solheim and Kjeldseth-Moe, 1987).

One problem with the system is that no orbital period has been detected (Solheim, 1988). The first attempt of high speed photometry was done by Robinson and Faulkner (1972) with the lick 120-inch telescope. No sinusoidal variations with a semiamplitude of $230 \mathrm{~km} \mathrm{~s}^{-1}$ were detected at the photometric period of $1051 \mathrm{~s}$.

Spectra were also taken by Voikhanskaya (1982), at the prime focus of the $6 \mathrm{~m}$ telescope. No significant changes in position or intensity of the He lines with the photometric period were observed, but she noted secular changes in the line profile. 
Our time resolved observations of $\mathrm{AM} \mathrm{CV}_{\mathrm{n}}$ have been carried out with the highest spectral and time resolution to date. This allows us to better estimate the limit of the radial velocity periodic variations and to study the line changes at different time scales.

\section{OBSERVATIONS}

The observations were made during three nights, 3th - 5th February 1987, at the Cassegrain focus of the $2.5 \mathrm{~m}$ Isaac Newton telescope at the Observatorio del Roque de los Muchachos, La Palma, Canary Islands. The spectrograph used was the IDS (Intermediate Dispersion Spectrograph) with the IPCS camera. The spectra covered the range $3950-4450 \AA$ with a spectral resolution of $0.25 \AA$ per channel. We took 485 short exposures of one minute duration each, in order to allow a coadding in phase for any desired period. As the detector is photon-counting limited, it allowed us to make short exposures without degrading the $\mathrm{S} / \mathrm{N}$ of the individual spectra.

\section{SPECTROSCOPIC VARIABILITY}

The data reduction process was carried using STARLINK programmes. For every selected period we added the spectra in eight phase intervals. Cross-correlations between the resultant spectra were made searching for Doppler shifts, in addition to a search for variations in equivalent width for the three absorption He I lines within our range ( $4026 \AA, 4144 \AA, 4388 \AA$ ). This period searches were made for different periods arising from high-speed photometric studies: $525 \mathrm{~s}, 1011 \mathrm{~s}, 2022 \mathrm{~s}$ and $1051 \mathrm{~s}$.

In a preliminary analysis we do not find significant changes which could be interpreted as orbital variations at these periods. However, changes in both depth and profile of the He I lines are found for all the searched periods. As an example, Figure 1 shows the behaviour of two of the He I lines for the $1051 \mathrm{~s}$ period. This implies that AM CVn presents spectral variations in time intervals as short as a few minutes.

As we did not find a clear periodicity, we searched for time variations adding spectra within time intervals from 15 minutes to one night. Figure 2 shows the added spectra for every hour in three nights. Variations in the asymmetry and depth of the He I lines are noticeable above the noise. A specially interesting event seems to have occurred at the end of the 3-4 Feb. night, with sudden intensity changes at the longer wavelength end of our spectral window, including the He I $4388 \AA$ line. A more detailed analysis will be published elsewhere. 

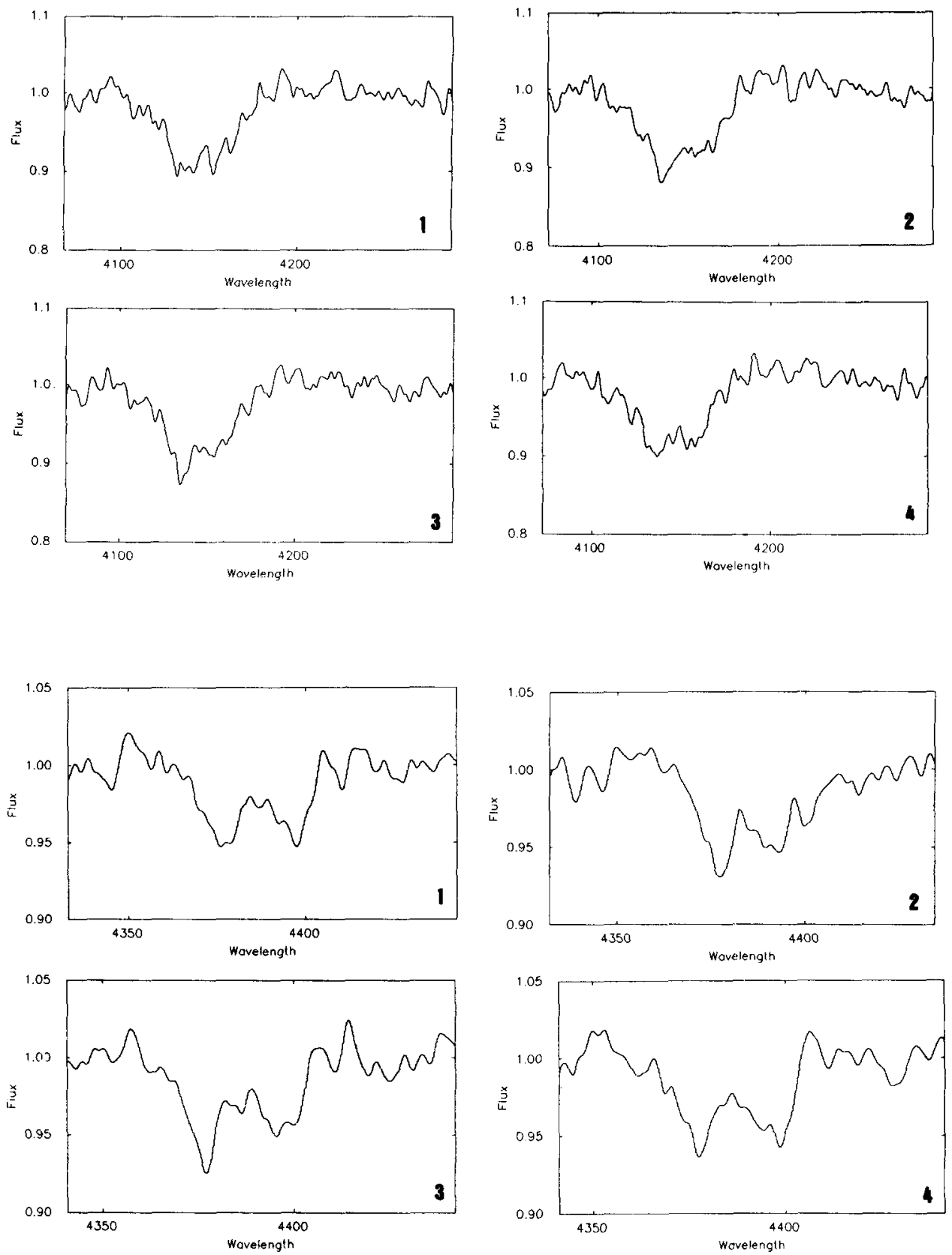

Figure 1: a) Line He I $4144 \AA$ with spectra coadded in phase intervals: $0.875-$ $0.125(1) ; 0.375-0.625(2) ; 0.12-0.375(3) ; 0.625-0.875(4)$. b) The same for the He I 4388 Å line. 
Faulkner, J., Flannery, B., and Warner, B.: 1972, Ap. J. (Letters) 125, L79. Krzeminski, V.: 1971, Remeis-Sternw. Bamberg 9, 178.

Robinson, E.L. and Faulkner, J.: 1972, Ap. J. (Letters) 200, L23.

Smak, J.: 1967, Acta Astron. 17, 225.

Solheim, J.-E.: 1988, IAU Coli. \# 114 .

Solheim, J.-E. and Kjeldseth-Moe, 0.: 1987, Astrophys. Space Sci. 131, 785. Voikhanskaya, N.F.: 1982 , Sov. Astron. 26, 558.

Warner, B. and Robinson, E.L.: 1972, Mon. Not. R, astro. Soc. 159, 101.

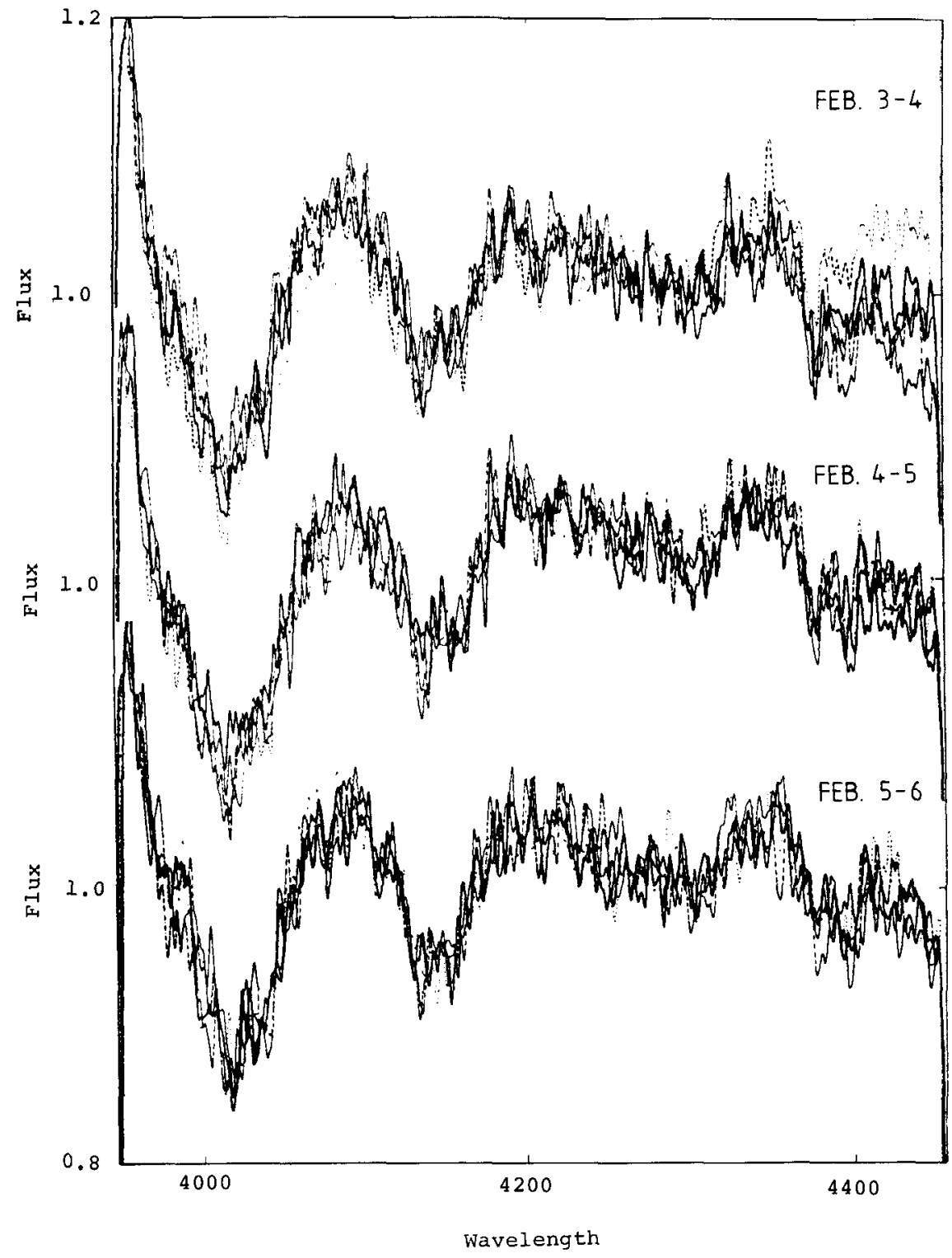

Figure 2: Added spectra in one hours interval for each night. 